\title{
RELACIÓN ENTRE CONDUCTAS PROSOCIALES Y PARTICIPACION EN GRUPOS ONLINE EN JOVENES CON DISCAPACIDAD MOTORA
}

\section{RELATIONSHIP BETWEEN PROSOCIAL BEHAVIOUR AND PARTICIPATION IN ONLINE SUPPORT GROUPS FOR YOUNG PEOPLE WITH MOTOR DISABILITY}

Raquel Suriá Martínez

Universidad de Alicante

\section{Abstract}

Objectives. The objectives of this study identify prosocial behavior of motor young users of online support groups depending on the participation in these virtual spaces. In addition, to identify significant differences in prosocial behavior according to the gender, age and grade of severity of disability. Method. A total of 114 young people with motor disability participated $(65,8 \%$ active participants users and $34,2 \%$ no active participants users). Aged 20 y 35 ( $M=28,22 ; S D=4,02)$. They completed the Prosocialness Scale for Adults (2005). Results. The results indicated more developed prosocial behavior in young participants users of online support groups. The results indicate that females score significantly higher than males on all dimensions of prosocial behavior. Moreover, the scores in this construct increase progressively with age. Conclusion. The results of this study can be useful as they highlight that online support groups could help to design prosocial behavior programs for young with motor disability.

Keywords: young people, motor disability, virtual support groups, prosocial behavior.

\section{Resumen}

Objetivos. Los objetivos del presente estudio identifican la conducta prosocial de los jóvenes usuarios de los grupos de apoyo online en función del nivel de participación en estos espacios virtuales. Asimismo, se examina si existen diferencias estadísticamente significativas en la conducta prosocial en función del sexo, edad y grado de severidad de discapacidad. Método. Participaron 114 jóvenes con discapacidad motora $(65,8 \%$ usuarios participantes activos y un 34,2\% usuarios participantes pasivos). El rango de edad fue 20 y 35 años $(M=28,22 ; D T=4,02)$. Contestaron la Escala para la Medición de la Conducta Prosocial en Adultos (2005). Resultados. Los resultados indicaron una conducta prosocial más desarrollada en los jóvenes usuarios participantes de los grupos de apoyo online. Los resultados indican que las mujeres puntúan significativamente más que los varones en todas las dimensiones de conducta prosocial. Además, con la edad aumentan progresivamente las puntuaciones en este constructo. Conclusión. Los resultados de este trabajo pueden resultar útiles, pues resaltan que la participación en los grupos de apoyo online podría contribuir en el diseño de programas que potencien la conducta prosocial de los jóvenes con discapacidad motora.

Palabras clave: jóvenes, discapacidad motora, grupos de apoyo virtuales, prosocialidad. 
Desde hace algunos años, internet ha irrumpido en la vida cotidiana de un amplio sector de la población, posibilitando nuevas formas de interacción entre sus usuarios. Uno de los recursos que ofrece la red son los grupos de apoyo para cuestiones relacionadas con la salud (Mercado-Martínez y Urias-Vázquez, 2014; Suriá y Beléndez, 2011; Villanueva, 2011). Estos grupos, son unos espacios ideales para un número considerable de afectados, y están dirigidos a tratar multitud de temas relacionados con la salud como por ejemplo, puede ser el caso de los grupos de apoyo para jóvenes con discapacidad motora (Augé y Escoin, 2003; Suriá, 2015).

De esta forma, además de los beneficios de los grupos de apoyo tradicionales, los usuarios de estos grupos, se benefician de otras singularidades que están disponibles en la red pero no en los grupos cara a cara. Así, por ejemplo los usuarios de grupos en línea pueden acceder al grupo a cualquier hora del día o de la noche sin tener una hora fijada como en los grupos cara a cara y sin tener que desplazarse (Campbell y Wright, 2002; Finfgeld, 2000; Weinberg, Schmale, Uken y Wessel, 1995). Esta flexibilidad de accesibilidad en la red es especialmente útil para las personas cuya discapacidad no les permite participar en las reuniones fijadas. Este sería el caso de los grupos de jóvenes con discapacidad motora, posiblemente, su movilidad reducida limite o dificulte asistir de en una manera regular a los grupos tradicionales (Salem, Bogat y Reid, 1997; Suriá, 2015).

En otros estudios se ha puesto de manifiesto que no sólo las personas con determinada tipología de discapacidad prefieren este recurso como forma de interacción, sino que algunos de ellos únicamente utilizan esta vía por encontrarla como la mejor forma de desenvolverse y sentirse apoyados (Cummings, Sproull y Kiesler, 2002; Davison, Pennebaker y Dickerson, 1997; Mickelson, 1997). En esta línea, Cummings et al. (2002), estudiaron en una muestra al azar de un grupo de autoayuda en línea para personas con discapacidad auditiva. Dos factores predijeron la satisfacción con el grupo: uno era la pérdida de audición y otro la falta de apoyo real en su entorno habitual. La participación más activa entre los miembros del grupo fue asociada con haber encontrado más beneficios en el grupo así como un sentimiento más fuerte de ser de la comunidad.

En cualquier caso, y con independencia de la infinidad de grupos de apoyo online existentes, se puede afirmar que todos tienen la misma finalidad, se agrupan para ofrecer ayuda a través de la acción de compartir sus experiencias personales vividas y de intercambiar conductas altruistas.

Así, la base que motiva a los miembros de los grupos de apoyo a formar parte de estas comunidades se encuentra en el comportamiento altruista compartido, conformado fundamentalmente por la interacción social y el intercambio de apoyo emocional, informacional y práctico, características fundamentales de las conductas prosociales (Eisenberg et al., 2006; inglés et al., 2015; Kilillea, 1976).

La conducta prosocial puede ser definida como todo aquel comportamiento social, positivo, de carácter voluntario y beneficioso para los demás (Eisenberg, Fabes y Spinrad, 2006; Martorell, González, Aloy, y Ferris 1995). Así, forma parte de la personalidad, comprendiendo acciones de ayuda, cooperación e intercambio y altruismo en las relaciones afectivas (Brown y Ferris, 2004; Fehr y Fischbacher, 2003), en el cumplimiento de las normas sociales (Caprara y Pastorelli, 1993; Lim, Khoo y Wong, 2007) y fundamental en el funcionamiento social y desarrollo psicológico del individuo (Hardy, Carlo y Roesch, 2010). Así, las pruebas indican que la conducta prosocial ha sido asociada de forma positiva con diferentes variables socioemográficas como el sexo (Calvo, González y Martorell, 2001; Redondo-Pacheco y Guevara-Melo, 2012), la edad (Carlo, Crockett, Randall y Roesch, 2007; Putnam, 2000) y el nivel educativo (Brown y Ferris, 2004; Magendzo, Toledo y Gutiérrez, 2013).

Otros atores han asociado la conducta prosocial a factores relacionados a la personalidad, como el autocontrol (Caprara y Pastorelli, 1993; Eisenberg et al., 2006), emociones positivas (Escrivá, García y Navarro, 2002) procesos cognitivos y razonamiento (Lemos y Richaud de Minzi, 2010), estabilidad emocional (Bekkers, 2006), confianza (Uslaner, 2002), autoconcepto (Garaigordobil, Cruz y Pérez, 2003) y autoeficacia social (Caprara y Steca, 2005), constructos necesarios para el afrontamiento exitoso de situaciones complejas, como vivir con discapacidad motora.

Centrándonos en el papel relevante que cobra el intercambio de conductas prosociales en los grupos de autoayuda online dirigidos a la discapacidad, todavía se hace más importante su fomento en personas que conforman etapas tempanas, como el colectivo de 
jóvenes con discapacidad. Sin embargo, si se echa una mirada a la literatura sobre los grupos de apoyo online dedicados a la discapacidad, la mayor parte de estas investigaciones han analizado la relación entre los usos de estos entornos virtuales y el apoyo social en función de la edad (Jiménez, de Ayala-López y García, 2013), del género (Espinar-Ruiz y González-Río, 2009), así como a grupos con diferentes entidades diagnósticas (Suriá y Beléndez, 2011) o con enfermedades específicas (Alarcón-Fernández et al., 2011), no existiendo estudios que analicen las conductas prosociales en el colectivo de jóvenes con discapacidad motora. Por tanto, uno de los objetivos de este trabajo pretende conocer la posible relación entre la pertenencia a estos grupos y las conductas prosociales que tienen los jóvenes con discapacidad participantes de los grupos de apoyo online.

Por otra parte, una de las brechas en el estudio del desarrollo y funcionamiento de los grupos de apoyo virtuales es la visibilidad y participación e intercambio de apoyo. Así, una de las características únicas de estos espacios es la posibilidad de acceder a su contenido sin necesidad de participar en ellos de manera activa. De forma que, para muchas personas el apoyo que se recibe en estos grupos virtuales tiene un carácter vicario ya que, sin tener que desvelar su problemática particular, pueden beneficiarse de las experiencias compartidas por los participantes activos del grupo, recibiendo ayuda y no aportando ningún apoyo al resto de miembros (Preece, Nonnecke y Andrews, 2004).

A estas personas que entran y que su participación no es activa se les conoce con el nombre de "lurkers" o comúnmente "mirones" ya que no participan en las discusiones y simplemente se dedican a leer, y de algún modo beneficiarse de los mensajes de apoyo del grupo.

Sobre ellos, han sido diversas las indagaciones efectuadas para conocer el sentido que tiene para estos individuos permanecer pasivos (Nonnecke, 2000; Nonnecke y Preece, 1999). Entre los motivos de la falta de participación activa se ha citado el preferir permanecer en el anonimato, el temor a ser ridiculizado por miembros más agresivos, la imposibilidad de aportar información por razones contractuales o de secreto profesional, disponer de poco tiempo para participar en grupos en línea, la creencia de no tener nada valioso que aportar, que carecen de pertenencia al grupo o se mantienen alejados dentro del mismo, carecer de intenciones altruistas, etc.

Por tanto, si en este trabajo se parte de la hipótesis de que los miembros de estos grupos intercambian conductas prosociales con su apoyo y participación, en este contexto, no tendría sentido catalogar el comportamiento de los lukers con la conducta prosocial, puesto que ni participan, ni ofrecen, ni intercambian ningún tipo de apoyo, de forma que, ¿es posible que la participación en los grupos de apoyo online guarde relación con la conducta prosocial?, en otros términos, ¿qué hace que unas personas participen más que otras?, ¿es posible que se deba a qué los miembros que más participan tengan comportamientos más prosociales que los usuarios que no participan?

Basándonos en el auge de estos grupos y con el propósito de profundizar en el estudio de la participación diferencial y su asociación con la conducta prosocial intercambiada en los grupos de apoyo online dirigidos a jóvenes con discapacidad motora. Este objetivo se desglosa en los siguientes objetivos específicos:

1. Conocer el nivel de participación de los participantes con discapacidad que utilizan los grupos de apoyo online dirigidos a este sector. Esto se examina en función del sexo, edad y grado de discapacidad.

2. Examinar la conducta prosocial en una muestra de usuarios de los grupos de apoyo dirigidos a jóvenes con discapacidad en función del nivel de participación. De forma complementaria, se analiza en función del sexo, edad y grado de discapacidad.

3. Valorar si existe relación entre la conducta prosocial y la participación, el sexo, la edad y el grado de discapacidad.

\section{MÉTODO}

\section{Participantes}

El estudio se llevo a cabo sobre una muestra intencional -por motivos de accesibilidad- de 114 jóvenes con discapacidad motora, todos ellos procedentes de diversas asociaciones dedicadas a personas con 
discapacidad, usuarios de los grupos de apoyo online. Inicialmente, la población de estudio estaba conformada por 164 jóvenes, sin embargo, de estos, fueron 114 los que se dispusieron a participar (ver tabla 1), correspondiendo el 53,5\%, al género femenino y el $46,5 \%$ al masculino, con edades comprendidas entre 20 y 35 años $(M=28,22 ; D T=4,02)$.

Tabla 1. Perfil sociodemográfico

\begin{tabular}{|c|c|c|c|}
\hline Perfil sociodemográfico & & $\mathrm{N}$ & $\%$ \\
\hline \multirow[b]{2}{*}{ Participación } & Nunca o poco & 39 & 33.6 \\
\hline & $\begin{array}{l}\text { Algo, } \\
\text { frecuentemente }\end{array}$ & 77 & 66.4 \\
\hline \multirow{2}{*}{ Sexo } & Mujer & 61 & 53,5 \\
\hline & Varón & 53 & 46,5 \\
\hline \multirow{3}{*}{ Edad } & $18-23$ & 29 & 26,7 \\
\hline & $24-29$ & 40 & 35,1 \\
\hline & $30-35$ & 45 & 38,3 \\
\hline \multirow{3}{*}{$\begin{array}{l}\text { Grado de severidad de } \\
\text { discapacidad }\end{array}$} & Menos del 33\% & 28 & 24,6 \\
\hline & Entre 33 y $65 \%$ & 42 & 36,8 \\
\hline & Más del 65\% & 44 & 38,6 \\
\hline \multirow{3}{*}{$\begin{array}{l}\text { Participación con } \\
\text { mensajes en el grupo de } \\
\text { apoyo }\end{array}$} & Nunca o poco & 39 & 34,2 \\
\hline & Algo o bastante & 75 & 65,8 \\
\hline & Total & 114 & 100,0 \\
\hline
\end{tabular}

\section{Instrumentos}

Cuestionario sociodemográfico; se diseñó un cuestionario ad hoc para la recogida de los datos sociodemográficos: género, edad, tipología, grado de severidad de la discapacidad de los participantes y participación en el grupo de apoyo (el criterio para averiguar el nivel de participación fue a través de la contribución de mensajes enviados).

Para medir la conducta prosocial de los participantes se utilizó la Escala para la Medición de la Conducta Prosocial en Adultos (Prosocialness Scale for Adults [PSA] de Caprara, Steca, Zelli y Capanna, 2005). Es una escala de dieciséis ítems, Tipo Likert con un formato de cinco alternativas de respuesta ( $1=$ nunca, 2 = pocas veces, $3=$ algunas veces, 4= a menudo, 5= habitualmente), en función de la frecuencia con que se den cada una de las conductas descritas. Cada ítem refleja comportamientos y sentimientos que pueden ser incluidos en cuatro factores: de altruismo, empatía, de compartir y de cuidar.
El criterio de rangos de conducta prosocial queda establecido en tres niveles en función de la puntuación mínima (20) y máxima (80). Los valores entre 61 y 80 indicarían un elevado nivel de conducta prosocial; entre 41-60, conducta prosocial moderada; y valores entre 20 y 40 , escasa conducta prosocial.

Se ha seleccionado este instrumento por ser breve (requiere aproximadamente 10 minutos su cumplimentación), específico (mide varias dimensiones de prosocialidad) y por sus adecuadas propiedades psicométricas $(\square=.92$ y varianza explicada de1 $68 \%)$.

Finalmente, para determinar las propiedades psicométricas del cuestionario en la muestra de este estudio, se calculó la validez a través la técnica del Análisis Factorial Exploratorio de Componentes Principales (Díaz de Rada Igurquiza, 2002), seguido de rotación Varimax para conseguir una mejor comprensión de la matriz resultante, quedando el $64.07 \%$ de la varianza explicada por la escala (25.93\% de varianza explicada para el primer factor, el 15.50\% quedó explicado por el segundo factor, $14.07 \%$ para el tercer factor y el último factor por el 8.56\%). Para analizar la fiabilidad del cuestionario se realizó una evaluación de la consistencia interna mediante el coeficiente Alfa de Cronbach, la cual indicó una consistencia adecuada ( $\square=.76$ ). Siguiendo el criterio establecido por Nunnally y Bernstein, (1995) se consideraron adecuados índices situados entre 0,70 y 0,80 para el cuestionario total.

\section{Procedimiento}

El cuestionario utilizado se alojó en un espacio de la empresa "Google" específicamente para la línea de investigación a la que se accede a través de un enlace y en el que se pedía la colaboración de los jóvenes con discapacidad motora. Para dar a conocer el enlace entramos en un espacio virtual dedicado al ámbito de la discapacidad, disponible en Facebook destinado a temas de accesibilidad universal desarrollado por el observatorio de la discapacidad del Centro de apoyo al estudiante de la universidad de Alicante. A su vez, se colocó el enlace en algunos espacios de la red social Facebook y Twitter creados por diferentes asociaciones dirigidas a personas con discapacidad motora en los que se explicó a través de un mensaje el objetivo, solicitando su colaboración para que cumplimentaran el cuestionario. Finalmente, los investigadores tenían vinculación directa con la Federación de ASPAYM 
(Asociación de Lesionados Medulares y Grandes Discapacitados Físicos) y con algunos jóvenes con discapacidad motora, con éstos, se utilizó el muestreo "bola de nieve", dando a conocer primero el objeto de la investigación y el enlace para acceder al cuestionario y posteriormente, solicitándoles que dieran a conocer la investigación a otros jóvenes con discapacidad motora para su colaboración. A continuación, se procedió al proceso de recogida de datos. La recopilación de los cuestionarios se llevó a cabo durante 2 meses.

\section{Análisis estadísticos}

Para examinar la frecuencia en participación en función del sexo, edad y grado de discapacidad se utilizó la prueba chi cuadrado $\left(\chi^{2}\right)$.

Para comprobar si la escala se adecuaba a los requerimientos de este estudio se comprobaron sus propiedades psicométricas en la muestra. Para ello, se utilizó el Análisis Factorial con Componentes Principales (AFECP), previa aplicación del test de esfericidad de Barlett y la medida de adecuación muestral KMO. La fiabilidad se comprobó a través del coeficiente Alfa de cronbach.

Para conocer si existían diferencias a nivel estadísticamente significativo en las puntuaciones de la escala y sus factores se utilizó la prueba $t$ para las variables dicotómicas (sexo y nivel de participación). Para las variables de más de 2 grupos (edad y grado de discapacidad), se utilizó el análisis de varianza. Posteriormente se realizaron pruebas post hoc para identificar entre qué grupos se encontraban las diferencias. Se utilizó el método de Scheffé al no estar cada grupo compuesto por el mismo número de participantes.

Finalmente, con el objetivo de deslindar la asociación de la conducta prosocial con las variables independientes posiblemente relacionadas (sexo, edad, grado de la discapacidad y nivel de participación), se calculó el coeficiente de correlación de Pearson para evaluar si existía relación entre las variables y se aplicó un modelo de regresión lineal múltiple. Para cumplir los requisitos del modelo, los datos se transformaron según el modelo de transformaciones potenciales desarrollado por Box y Cox (1964). Se utilizó esta prueba puesto que permite estimar la transformación potencial más adecuada cuando no hay una razón a priori para elegir un modelo concreto de transformación.

\section{RESULTADOS}

Con respecto al primer objetivo, conocer el perfil de uso de los participantes con discapacidad que utilizan los grupos de ayuda online dirigidos a este sector se observa un $34,2 \%$ indica participar poco o nada mientras que el $65,8 \%$ muestra participar habitualmente.

Tabla 2. Participación activa de los usuarios en los grupos de apoyo online según sexo, edad y grado de discapacidad

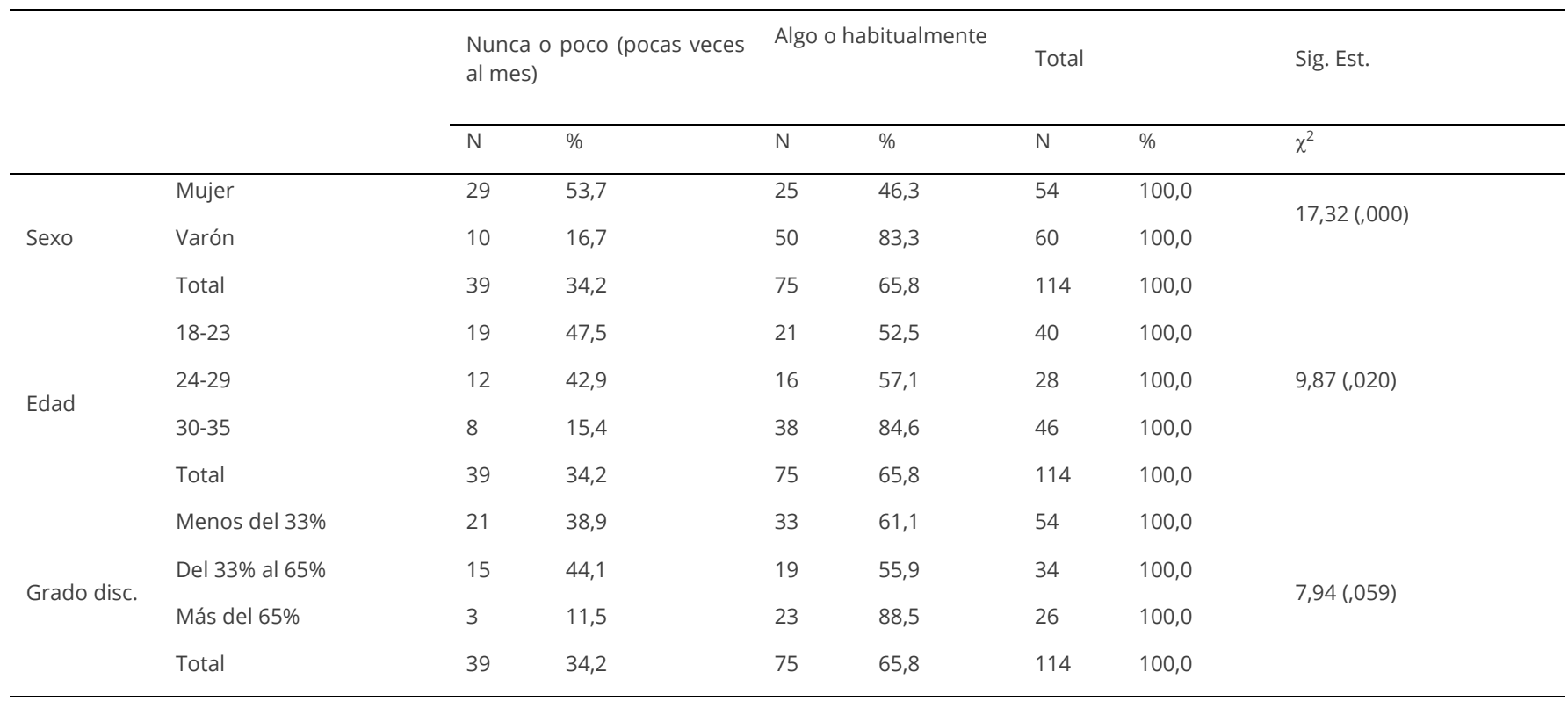


Al examinar si existe relación entre el nivel de participación en función de las variables sociodemográficas, se observaron diferencias estadísticamente significativas en función del sexo $\left(\chi^{2}(1)\right.$ $=17,322, p<, 001)$, así como en función de la edad $\left(\chi^{2}(2)\right.$ $=9,871, p<, 05)$.

En relación al segundo objetivo, examinar la conducta prosocial en una muestra de usuarios de los grupos de apoyo dirigidos a jóvenes con discapacidad, se observa que, en general, indican un nivel elevado de conducta prosocial $(M=49,40, D . T=7,11)$. Al examinar la conducta prosocial en función del nivel de participación, las puntuaciones medias de la escala global indicaron ser superiores en los usuarios que frecuentan participar tanto en la escala global $\left(t_{(114)}=113,16, p<, 000\right)$, como en
Ios factores relativos al Altruismo $\left(t_{(114)}=113,58, p<, 000\right)$, al factor referente a Compartir, $\left(t_{(114)}=6113,16, p<, 000\right)$, $\mathrm{y}$ al Apoyo $(t(114)=6113,16, p<, 000)$, (Tabla 3).

Con respeto a la conducta prosocial en función de las variables sociodemográficas (sexo, edad y grado de discapacidad, Tabla 2), se observan diferencias estadísticamente significativas en función del sexo tanto en la escala global $\left(F_{(2,113)}=107,22, p<, 001\right)$, como en los cuatro factores de la escala. Asimismo, se observan diferencias estadísticamente significativas en la escala global de conducta prosocial en función de la edad tanto en la escala $\left(F_{(2,111)}=6,087, p<, 05\right)$, como en los factores que la conforman. No se observan diferencias estadísticas según el grado de discapacidad $\left(F_{(2,113)}=\right.$ $6,677, p=, 072)$.

Tabla 3. Puntuaciones medias y Desviaciones típicas de conducta prosocial según participación, sexo, edad y grado de discapacidad

\begin{tabular}{|c|c|c|c|c|c|c|c|c|c|c|c|}
\hline & \multicolumn{3}{|c|}{ Escala Total } & \multicolumn{2}{|c|}{ Altruismo } & \multicolumn{2}{|c|}{ Compartir } & \multicolumn{2}{|l|}{ Apoyo } & \multicolumn{2}{|l|}{ Empatía } \\
\hline & $N$ & $M$ & $D . T$ & $M$ & D.T & $M$ & D.T & $M$ & $D . T$ & $M$ & $D . T$ \\
\hline Escala total & 114 & 49,40 & 7,11 & 12,54 & 2,92 & 8,62 & 2,31 & 7,66 & 1,96 & 10,89 & 2,46 \\
\hline \multicolumn{12}{|c|}{ Nivel de participación } \\
\hline Nunca o poco & 39 & 44,85 & 5,62 & 10,98 & 2,38 & 6,64 & 2,05 & 6,26 & 1,89 & 9,95 & 1,66 \\
\hline Algo o bastante & 75 & 51,35 & 6,05 & 15,97 & 2,67 & 9,30 & 2,23 & 9,78 & 1,75 & 10,40 & 2,48 \\
\hline \multirow[t]{2}{*}{$t$ (sig.) } & & 113,16 & $(, 000)$ & 113,58 & $(, 000)$ & 113,12 & $(, 000)$ & 107,05 & $(, 000)$ & 113,99 & $(, 110)$ \\
\hline & & & & & Sexo & & & & & & \\
\hline Varón & 54 & 46,07 & 6,76 & 12,46 & 2,87 & 7,06 & 2,37 & 6,67 & 1,89 & 8,83 & 2,28 \\
\hline Mujer & 60 & 49,58 & 6,93 & 13,60 & 2,64 & 7,97 & 3,17 & 9,45 & 1,96 & 10,85 & 2,20 \\
\hline t(sig.) & & 107,22 & $(, 000)$ & 108,51 & $(, 000)$ & 108,39 & $(, 049)$ & 112,70 & $(, 030)$ & 110,64 & $(, 000)$ \\
\hline $18-23$ & 40 & 41,78 & 7,17 & 12,75 & 3,06 & 6,95 & 2,50 & 6,48 & 2,01 & 9,40 & 2,82 \\
\hline $24-29$ & 34 & 42,21 & 7,48 & 13,29 & 2,71 & 7,50 & 2,27 & 6,71 & 1,61 & 9,50 & 2,27 \\
\hline $30-35$ & 40 & 49,44 & 6,30 & 15,44 & 2,91 & 9,25 & 1,81 & 9,78 & 1,93 & 10,22 & 2,20 \\
\hline \multirow[t]{2}{*}{$F$ (sig.) } & & 6,087 & $(, 001)$ & 2,869 & $(, 040)$ & 1,972 & $(, 422)$ & 4,160 & $(, 008)$ & 2,897 & $(, 038)$ \\
\hline & & & & \multicolumn{4}{|c|}{ Grado de discapacidad } & & & & \\
\hline Menos del 33\% & 26 & 48,37 & 6,99 & 11,33 & 3,03 & 7,04 & 2,47 & 6,96 & 2,15 & 9,76 & 2,71 \\
\hline Del 33\% a 65\% & 34 & 48,06 & 7,50 & 10,94 & 2,86 & 7,56 & 2,15 & 7,03 & 1,83 & 9,35 & 2,23 \\
\hline Más del 65\% & 54 & 49,64 & 6,42 & 11,93 & 2,69 & 9,50 & 2,04 & 9,39 & 2,53 & 10,89 & 2,09 \\
\hline$F$ (sig.) & & 6,677 & $(, 072)$ & 4,151 & $(, 078)$ & 3,932 & $(, 062)$ & ,460 & $(, 059)$ & 3,373 & $(, 066)$ \\
\hline
\end{tabular}

Finalmente (Tabla 4), se observa una relación positiva entre la conducta prosocial con el nivel de participación $(\eta=, 733, p<, 001)$, con el sexo $(456)$ y la edad $(\eta=, 630, p<, 001)$. Al incluir las cuatro variables en un modelo de análisis de regresión múltiple (Tabla 4), los datos reflejan un valor de ANOVA estadísticamente significativo $F_{(4,111)}=3,308, p<, 001$, con unos coeficientes estandarizados estadísticamente significativos $(p<, 05)$, para el nivel de participación $(, 713)$ y la edad $(, 128)$. 
Tabla 4. Coeficientes y niveles de significación para las variables incluidas en el modelo

\begin{tabular}{llllll}
\hline & B & Error típico & Beta & $t$ & $p$ \\
\hline Constante & 21,89 & 1,71 & & 12,74 &, 000 \\
Participación & 10,18 & 1,23 &, 71 & 8,23 &, 000 \\
Edad &, 85 & 0,52 &, 12 & 1,61 &, 048 \\
Sexo &,- 29 & 1,17 &,- 02 &,- 24 &, 054 \\
Grado disc. &,- 27 & 0,68 &,- 03 &,- 40 &, 685
\end{tabular}

\section{DISCUSIÓN}

Con el presente trabajo se pretende cuantificar el nivel de conducta prosocial que reflejan los jóvenes con discapacidad en los grupos de apoyo online en función del nivel de participación. Asimismo, valorar la posible asociación entre este constructo con la participación y algunas variables sociodemográficas como el sexo, edad y grado de discapacidad de los jóvenes usuarios de estos entornos online.

Así, tal y como se comprueba en los resultados del primer objetivo, se observa que la mayoría de jóvenes son participantes activos de este recurso de apoyo. Por tanto, aunque es evidente que estos espacios son utilizados por usuarios sin ningún tipo de discapacidad, para algunos jóvenes con determinadas características, como vivir con discapacidad motora, es un recurso en el que participan con asiduidad (Eden y Seiman, 2011; Suriá, 2015; Zubillaga-del Río y Alba-Pastor, 2013).

Esto se reafirma en mayor medida entre las mujeres, y entre los participantes de más edad. En este sentido, diferentes autores señalan que el patrón diferencial encontrado en la participación en los grupos de internet en función del sexo se basan en los diferentes motivos de uso, así, los hombres están más centrados en la participación en temas de ocio y entretenimiento, mientras que las mujeres se centran en cuestiones más funcionales relacionadas con temas emocionales y de salud (Espinar-Ruiz y González, 2009; Herring y Androutsopoulos, 2015). Del mismo modo, los usuarios de estos entornos difieren $n$ la participación según la edad, corroborando esto los resultados de otros autores que afirman que los temas preferentes entre los jóvenes más mayores son temas relacionados con la información y la salud (Herrero, Meneses, Valiente y Rodríguez, 2004).
En relación al segundo objetivo, conocer la conducta prosocial en una muestra de jóvenes usuarios de los grupos de apoyo dirigidos a la discapacidad motora, se observa que las puntuaciones medias en la escala reflejan una conducta prosocial bastante adecuada.

En este sentido, el hándicap de vivir con discapacidad, puede ser un factor que fomente el desarrollo de comportamientos prosociales. Así, el hecho de que la persona con discapacidad motora deba enfrentarse a infinidad de obstáculos en su día a día puede ser un factor que potencie la capacidad de ponerse en el lugar de los demás, su competencia emocional así como la habilidad de desarrollar conductas prosociales (Morales, Fernández, Infante, Trianes y Cerezo, 2010).

Al atender a las variables analizadas en el estudio, que pueden asociarse al desarrollo de la conducta prosocial, se observan los siguientes resultados:

Al examinar los resultados sobre el nivel de conducta prosocial en función de la participación de los usuarios, los datos reflejan que los usuarios que participan activamente mostraron un nivel elevado de conducta prosocial, mientras que los usuarios que no suelen participar indicaron un nivel moderado. Estos resultados indican que, en mayor menor grado, los participantes tienen desarrollado este constructo, sin embargo, la participación es más activa en los usuarios con conductas prosociales. Esto se releja en la escala global y en algunos factores, como en el factor 1, altruismo, factor 2, compartir y en el factor 3, apoyo.

Posiblemente, los usuarios que participan activamente, al involucrarse con el intercambio de mensajes, puedan tener más desarrollado el sentido de identidad con el grupo y mayor compromiso con los integrantes de estas comunidades de compartir sus experiencias que los usuarios que únicamente se limitan a leer los mensajes.

En referencia a esto, Lave y Wenger (1991), sugieren que los usuarios no participan activamente son más reacios a participar, a intercambiar apoyo y en definitiva, conductas prosociales por la noción de "Participación Periférica Legítima". Con este concepto, sostienen que la razón por la que los usuarios no participantes o lurkers deciden no escribir mensajes y solo leerlos se justifica porque éstos se dedican a observar las normas y la forma de escribir los mensajes de los otros miembros del grupo 
porque no tienen desarrolladas sus habilidades sociales y su capacidad de empatizar con otros. De esta forma, leer mensajes les permitiría aprender y ganar la confianza necesaria para expresarse en público con empatía, y de este modo, aprender a compartir sus experiencias y saber integrarse adecuadamente en el grupo o comunidad virtual (Correll, 1995; Lave y Wenger, 1991; Lindlof y Shatzer, 1998; Zhang y Storck, 2001).

Con respecto al desarrollo de conducta prosocial de los usuarios de los grupos de apoyo online en función del sexo, los resultados muestran que las mujeres tienen mayor nivel de conducta prosocial. Esto se refleja en todos los factores de la escala.

Estos resultados corroboran los obtenidos por diferentes estudios que revelan que las mujeres muestran niveles más altos de conducta prosocial que los varones (Calvo, et al., 2001; Inglés, Martínez-González y García-Fernández, 2015; Sánchez-Queija, Oliva y Parra, 2006). Así, en estos se argumenta que el patrón diferencial que las diferencias de conducta prosocial en función del género quedan justificadas por el tipo de demanda. En este sentido, los hombres tienden a ayudar a otros cuando se precisan acciones rápidas y de carácter material, sin embargo, las mujeres, desarrollan preferentemente comportamientos prosociales en contextos de relaciones sociales, realizando acciones de apoyo, cuidados o empatía. Este tipo de conducta prosocial se ajustaría al apoyo ofrecido en los grupos virtuales. En esta línea, Caprara y Steca (2005), señalan que estos resultados se originan por los estereotipos sociales y culturales que atribuyen a la mujer una mayor sensibilidad emocional, una mayor tendencia al cuidado y apoyo a los más débiles (ej., personas con discapacidad), una mayor capacidad para detectar sentimientos y señales de ayuda y una mayor preocupación por los aspectos sociales de la interacción y los sentimientos de los demás.

En referencia al desarrollo de la conducta prosocial en función de la edad, se observa en los resultados que los grupos de participantes de más edad puntúan más elevado tanto en la puntuación global como en los factores que conforman este constructo.

De acuerdo con la literatura relevante (Eisenberg, Cumberland, Guthrie, Murphy y Shepard, 2005; Eisenberg et al., 2002; Eisenberg, Zhou y Koller, 2001), la orientación prosocial parece ser una marca de la entrada en la madurez adulta, desarrollándose tanto en adolescentes como por jóvenes y culminando con la madurez. Esto es debido, en parte, a la madurez cognitiva que propicia el desarrollo de la empatía, del razonamiento moral, prosocial y de la capacidad de comprender los estados internos y externos de los demás (Eisenberg et al., 2005).

Finalmente, al atender a la relación de las variables que pueden asociarse al desarrollo de la conducta prosocial (participación, sexo, edad y grado de discapacidad), se observa que la participación, el sexo y la edad, guardan relación directa positiva con la conducta prosocial, no observándose asociación entre este constructo y el grado de discapacidad. En este sentido, el hecho de vivir esta experiencia, puede ser un factor que ayude a comprender situaciones complejas y experiencias similares entre los afectados, y por tanto, que no interfiera el grado de severidad de la discapacidad en el desarrollo de su conducta prosocial (Morales et al., 2010). Estos datos, ofrecen consistencia a la validez de los resultados obtenidos en el objetivo anterior consistente en examinar si los participantes difieren en la conducta prosocial según el grado de severidad de la discapacidad, los cuales reflejan que no se observan diferencias estadísticamente significativas en la conducta prosocial en función de esta variable.

Por tanto, como los resultados obtenidos reflejan, parece que, que la conducta prosocial de los jóvenes con discapacidad motora se relaciona con la participación en los grupos de apoyo online dirigidos a esta tipología de discapacidad, y por tanto, ahondar en esta asociación puede resultar interesante para el fomento de la prosocialidad. No obstante, se deben tener en cuenta algunas limitaciones de este trabajo. En primer lugar, el número de participantes es muy reducido y se limitan a población joven con discapacidad motora. Esto dificulta que sea posible realizar inferencias sobre si otras muestras obtendrían las mismas conclusiones. Por tanto, en futuras investigaciones es necesario aunar esfuerzos por obtener otras muestras de validación cruzada que repliquen los resultados. De la misma forma, los participantes de este estudio se ofrecieron voluntariamente para cumplimentar los cuestionarios, por lo que éstos podrían tener diferentes motivaciones para participar en comparación con los jóvenes que se mostraron reacias a participar. Asimismo, es lógico suponer que los participantes pertenecen a alguna asociación, esto puede conllevar que tengan desarrollado 
cierto nivel de prosocialidad. Estos aspectos sobreestimarían el grado de conducta prosocial y podrían sesgar la magnitud de algunas de las asociaciones detectadas. En futuras investigaciones se deberían controlar estos sesgos con objeto de incrementar la validez interna de los resultados. Por último, podría ser fructífera la comparación de la utilización de los grupos de apoyo online y el nivel de conducta prosocial de los jóvenes usuarios que no viven con este hándicap.

\section{REFERENCIAS}

Alarcón-Fernández, O., Alonso-Abreu, I., Carrillo-Palau, M., Nicolás-Pérez, D., Gimeno, A. Z., Ramos, L. y Quintero-Carrión, E. (2011). Uso de Internet entre los pacientes con enfermedades digestivas en un hospital general. Gastroenterología y Hepatología, 34(10), 667-671.

Augé, C. y Escoin, J. (2003). Tecnologías de ayuda y sistemas aumentativos y alternativos de comunicación en personas con discapacidad motora. F. Alcantud y FJ Soto (coords.), Tecnologías de ayuda en personas con trastornos de comunicación, 139-160.

Bekkers, R. (2006). Traditional and Health Related Philanthropy: The Role of Resources and Personality. Social Psychology Quarterly, 68(4), 349366.

Box, G. E. y Cox, D. R. (1964). An analysis of transformations. Journal of the Royal Statistical Society. Series B (Methodological), 211-252.

Brown, E. y Ferris, J. M. (2007). Social Capital and Philanthropy: An Analysis of the Impact of Social Capital on Individual Giving and Volunteering. Nonprofit and Voluntary Sector Quarterly, 36(1), 85-99.

Calvo, A. J., González, R. y Martorell, M. C. (2001). Variables relacionadas con la conducta prosocial en la infancia y adolescencia: personalidad, autoconcepto y género. Variables related to prosocial behaviour in childhood and adolescence: Personality, self-concept and gender. Infancia y aprendizaje, 24(1), 95-111.

Campbell, K. y Wright, K. B. (2002). On-line support groups: An investigation of relationships among source credibility, dimensions of relational communication, and perceptions of emotional support. Communication Research Reports, 19(2), 183-193.

Carlo, G., Crockett, L. J., Randall, B. A. y Roesch, S. C. (2007). A latent growth curve analysis of prosocial behavior among rural adolescents. Journal of Research on Adolescence, 17(2), 301-324.

Caprara, G. V., Steca, P., Zelli, A. y Capanna, C. (2005). A new scale for measuring adults' prosocialness. European Journal of Psychological Assessment, 21(2), 77-89.

Caprara, G. V., y Steca, P. (2005). Affective and social self-regulatory efficacy beliefs as determinants of positive thinking and happiness. European Psychologist, 10(4), 275.

Caprara, G. V. y Pastorelli, C. (1993). Early emotional instability, prosocial behavior, and aggression: some methodological aspects. European Journal of Personality, 7(1), 19-36.

Correll, S. (1995). The ethnography of an electronic bar: The Lesbian Cafe. Journal of Contemporary Ethnography, 24 (3), 270-290.

Cummings, J. N., Sproull, L. y Kiesler, S. B. (2002). Beyond hearing: Where the real-world and online support meet. Group Dynamics: Theory, Research, and Practice, 6(1), 78.

Davison, K.P., Pennebaker, J.W. y Dickerson, S.S. (2000). Who talks? The social psychology of illness groups. American Psychologist, 55(2), 205217.

Díaz de Rada Igurquiza, V. (2002). Análisis Factorial. En: Díaz de Rada Igurquiza V. Técnicas de análisis multivariante para investigación social y comercial (pp. 91-156). Madrid: RA-MA.

Eden, S. y Heiman, T. (2011). Computer mediated communication: Social support for students with and without learning disabilities. Educational Technology \& Society, 14, 89-97.

Espinar Ruiz, E. y González Río, M. J. (2009). Jóvenes en las redes sociales virtuales: un análisis exploratorio de las diferencias de género.

Eisenberg, N., Cumberland, A., Guthrie, I. K., Murphy, B. C. y Shepard, S. A. (2005). Age changes in prosocial responding and moral reasoning in adolescence and early adulthood. Journal of research on adolescence, 15(3), 235-260.

Eisenberg, N., Fabes, R.A y Spinrad, T. L. (2006). Prosocial development. En W. Damon y N. Eisenberg (Eds.), Handbook. of child psychology, Vol. 3: Social, emotional and personality development (pp. 646-718). Nueva York: John Wiley y Sons.

Eisenberg, N., Guthrie, I. K., Cumberland, A., Murphy, B. C., Shepard, S. A., Zhou, Q... y Carlo, G. (2002). Prosocial development in early adulthood: a longitudinal study. Journal of personality and social psychology, 82(6), 993.

Escrivá, M. V. M., García, P. S. y Navarro, M. D. F. (2002). Procesos cognitivos y emocionales predictores de la conducta prosocial y agresiva: la empatía como factor modulador. Psicothema, 14(2), 227 232.

Fehr, E. y Fischbacher, U. (2003). The Nature of Human Altruism. Nature, 425, 785-791.

Finfgeld, D. L. (2000). Therapeutic groups online: the good, the bad, and the unknown. Issues in mental health nursing, 21(3), 241-255.

Garaigordobil, M., Cruz, S. y Pérez, J. I. (2003). Análisis correlacional y predictivo del autoconcepto con otros factores conductuales, cognitivos y emocionales de la personalidad durante la adolescencia. Estudios de psicología, 24(1), 113-134.

Hardy, S. A., Carlo, G. y Roesch, S. C. (2010). Links Between Adolescents' Expected Parental Reactions and Prosocial Behavioral Tendencies: The Mediating Role of Prosocial Values. Journal Youth Adolescence, 39, 84-95.

Herrero, J., Meneses, J., Valiente, L. y Rodríguez, F. (2004). Participación 
social en contextos virtuales. Psicothema, 16(3), 456-460.

Herring y Androutsopoulos, J. (2015). 6 Computer-Mediated Discourse 2.0. The handbook of discourse analysis, 127.

Inglés, C. J., Martínez-González, A. E. y García-Fernández, J. M. (2015). Conducta prosocial y estrategias de aprendizaje en una muestra de estudiantes españoles de Educación Secundaria Obligatoria. European Journal of Education and Psychology, 6(1), 33-53.

Jiménez, A. G., de Ayala López, M. C. L. y García, B. C. (2013). Prácticas comunicativas de los adolescentes en las redes sociales: concienciación y exposición a riesgos online. In Espacios de comunicación: IV Congreso Internacional de la Asociación Española de Investigación en Comunicación (p. 962). Asociación Española de Investigación de la Comunicación.

Lave, J. y Wenger, E. (1991). Situated Learning: Legitimate Periperal Participation. Cambridge, UK: Cambridge University Press.

Lemos, V. N. y Richaud de Minzi, M. C. (2010). Construcción de un instrumento para evaluar el razonamiento prosocial en niños de 7 y 8 años: una versión pictórica. Universitas Psychologica, 9 (3), 879891.

Lim, K. M, Khoo, A. y Wong, M. Y. (2007). Relationship of Delinquent Behaviors to Prosocial Orientations of Adolescents, North American Journal of Psychology, 9, 183-188.

Lindlof, T. R. y Shatzer, M. J. (1998). Media ethnography in virtual space: Strategies, limits, and possibilities. Journal of Broadcasting \& Electronic Media, 42, 170-186.

Magendzo, A., Toledo, M. I. y Gutiérrez, V. (2013). Descripción y análisis de la Ley sobre Violencia Escolar: dos paradigmas antagónicos. Estudios pedagógicos, 39(1), 377-391.

Martorell, M.C, González, R., Aloy, M. y Ferris, M.C. (1995). Socialización y conducta prosocial. Revista Iberoamericana de Diagnóstico y Evaluación Psicológica, 1, 73-102.

Mercado-Martínez, F. J. y Urias-Vázquez, J. E. (2014). Hispanic American kidney patients in the age of online social networks: content analysis of postings, 2010-2012. Revista Panamericana de Salud Pública, 35(5-6), 392-398.

Mickelson, K. D. (1997). Seeking social support: Parents in electronic support groups. Culture of the Internet, 157-178.

Morales, F. M., Fernández, F. J., Infante, L., Trianes, M. V. y Cerezo, M. T. (2010). Eficacia de una intervención para incrementar apoyo social en adolescentes discapacitados motores a partir del voluntariado de estudiantes de educación secundaria. Revista Latinoamericana de Psicología, 41, 141-152.

Nonnecke, B. (2000). Lurking in email-based discussion lists, publicado en SCISM. London: South Bank University.

Nonnecke, B.y Preece, J. (1999). Shedding light on lurkers in online communities. En Buckner, K. (ed.), Ethnographic Studies in Real and Virtual Environments Inhabited Information Spaces and Connected Communities, Edinburgh, pp.123-128.
Nunnally, J.C. y Bernstein, I.J. (1995). Teoría psicométrica. Madrid: McGrawHill.

Redondo-Pacheco, J. y Guevara-Melo, E. (2012). Diferencias de género en la prevalencia de la conducta prosocial y agresiva en adolescentes de dos colegios de la ciudad de Pasto-Colombia. Revista Virtual Universidad Católica del Norte, 1(36), 173-192.

Preece, J., Nonnecke, B. y Andrews, D. (2004). The top 5 reasons for lurking: Improving community experiences for everyone. Special Issue of Computers in Human Behavior: An Interdisciplinary Perspective, 20(2).

Putnam, R. (2000). Bowling Alone: The collapse and revival of American Community. New York: Simon \& Schuster.

Salem, D. A., Bogat, G. A. y Reid, C. (1997). Mutual help goes on-line. Journal of Community Psychology, 25(2), 189-207.

Sánchez-Queija, I., Oliva, A. y Parra, Á. (2006). Empatía y conducta prosocial durante la adolescencia. Revista de Psicología Social, 21(3), 259-271.

Suriá, R. (2015). Jóvenes con discapacidad motora y redes sociales online, ¿ nuevos espacios para el desarrollo de habilidades sociales. Anuario de psicología/The UB Journal of psychology, 45(1), 71-85.

Suriá, R. (2016). Análisis comparativo del uso excesivo de móvil entre jóvenes con y sin movilidad reducida.

Suriá, R. y Beléndez, M. (2011). Grupos de apoyo virtuales dedicados a problemas de salud: estudio de su tipología y análisis de su representatividad. Anales de psicología, 27(1), 210-220.

Uslaner, E. M. (2002). The Moral Foundations of Trust. Cambridge, UK: Cambridge University Press.

Villanueva, F. L. (2011). Salud e internet: más allá de la calidad de la información. Revista española de cardiología, 64(10), 849-850.

Weinberg, N., Schmale, J. D., Uken, J. y Wessel, K. (1995). Computermediated support groups. Social Work with Groups, 17(4), 43-54.

Zhang, W. y Storck, J. (2001). Peripheral Members in Online Communities. Proceedings of AMCIS: Boston.

Zubillaga-del Río, A. y Alba-Pastor, C. (2013). La discapacidad en la percepción de la tecnología entre estudiantes universitarios. Comunicar: Revista Científica de Comunicación y Educación, 20(40), 165-172. 